\title{
Broadband push-pull power amplifier design methodology based on the GaN component base for high-performance nonlinear junction detectors
}

\author{
Vladimir Klokov ${ }^{1, *}$, Nikolay Kargin ${ }^{1}$, Alexander Garmash ${ }^{1}$, and Ekaterina Guzniaeva ${ }^{1}$ \\ ${ }^{1}$ National Research Nuclear University MEPHI, 115409 Kashirskoe shosse 31, Moscow, Russia
}

\begin{abstract}
The paper presents a description of design methodology for wide-band push-pull large-signal power amplifier based on GaN transistor with an output power of more than $10 \mathrm{~W}$ for high-performance Nonlinear Junction Detectors, which allows achieving optimal convergence of the theoretical model in practice, as well as increasing the efficiency of the power amplifier while maintaining a linear gain characteristic.
\end{abstract}

\section{Introduction}

The classical method to detect a hidden microelectronic devices, containing working or non-working elements with a non-linear I - V characteristic, is the method of emitting a polarized electromagnetic wave of a monochromatic (single-frequency) signal and receiving the second and third harmonic components of the echo signal. The emitted signal is modulated in amplitude, and the dependence of the amplitude of response signal on the amplitude of the probing signal is measured at the receivers, which gives additional information about the type of nonlinearity. The main problems of this approach is the small detection range due to the influence of the harmonics of emitted signal on the receiver input, as well as the inability to determine the type of device detected due to insufficient input data. One of the modern approaches in the field of detection of nonlinear junction is the method of sensing using broadband modulated radiation with a central frequency fc, a BW band and receiving and digitizing the echo signal, using high-speed ADCs, at the central frequencies $2 \mathrm{fc}$ and $3 \mathrm{fc}$. The use of a broadband modulated emitted signal allows, in addition to obtain the dependence of the microwave power on the amplitude of the echo signal, to also measure the nonlinear distortion of the received modulated echo signal, which greatly expands the possibilities of analysis to determine the type of device detected.

Studies shows that the use of emitted from a broadband modulated microwave signal can increase the detection range of hidden electronic devices. However, the development of such high-performance devices for detecting nonlinear transitions requires more complex configuration of both the high-frequency amplifier path of the transmitting signal and the antenna-feeder system:

- increased requirements for the width of the working band of the amplifier;

* Corresponding author: VAKlokov@mephi.ru 
- increased requirements for the linearity of the amplifier;

- the need to conduct the development of PA taking into account non-linear distortions, due to the use of a broadband modulated signal;

- decrease in the level of harmonic components of the broadband signal.

In the field of constructing output power amplifiers, it is to some extent limited by the lack of a sufficient nomenclature of the component base with the necessary power parameters. Silicon and gallium arsenide high-power microwave transistors have almost reached their limit due to the limitations of the electrophysical parameters of the initial semiconductor materials. To create a microwave component base and powerful amplifiers, the advantages of gallium nitride technology, which were reflected in previous studies [12], are especially important.

This paper presents the results of applying the design Methodology of a wideband pushpull large-signal power amplifier based on the GaN component base for high-performance devices for detecting nonlinear junction. The methodology reflects solutions to the problems of convergence of design results in the development of the Push-Pull topology. Comparison of two different topologies of PA is shown for the comparison of their characteristics.

\section{Object of research}

$\mathrm{GaN}$ transistor amplifier CG2H40025F manufactured by Cree Inc was used as an object of research [3]. To solve the tasks posed in the work, two topologies of the power amplifier were designed: a single-stage class A power amplifier and a push-pull power amplifier. Comparisons were made when the output characteristics were achieved: signal band: 3800 $4100 \mathrm{MHz}$, output power: at least 10 watts.

\section{Power amplifier design}

The amplification path for nonlinear junction detectors should operate in a linear or near to linear mode in order to minimize the excitation of the harmonic components of the probe signal. Also, amplifier circuit should provide sustainability and temperature stability, to ensure the operational characteristics of the product.

To solve this problem, a push-pull power amplifier based on a GaN transistor was proposed.

The main advantages of using push-pull topology are follows:

- a Class A linear output signal can be obtained when the efficiency of a Class B amplifier is achieved, but using two GaN transistors instead of one.

- push-pull topology assumes an ideal short circuit at the even harmonics of the main signal, which allows to increase the efficiency of the PA as applied to nonlinear junction detection devices.

However, there is a problem to achieve the stability and efficiency of PA made by the push-pull topology at frequencies above $2 \mathrm{GHz}$.

Figure 1 shows the proposed block diagram of PA using a push-pull topology for use in nonlinear transition detection devices.

The proposed block diagram contains signal division and addition schemes with an output phase difference of 180 degrees and output ports with an impedance of $25 \mathrm{Ohms}$, input and output circuits matching $25 \mathrm{Ohms}$, an output circulator, and low-pass filters for gate and drain power. In the proposed method, instead of filter-based matching circuits, that limit the amplifier performance for broadband applications, it is proposed to use a simplified circuit topology based on the proximity of the transistor load resistance to an output resistance of $25 \mathrm{Ohms}$ of the applied division and addition circuits with an output 


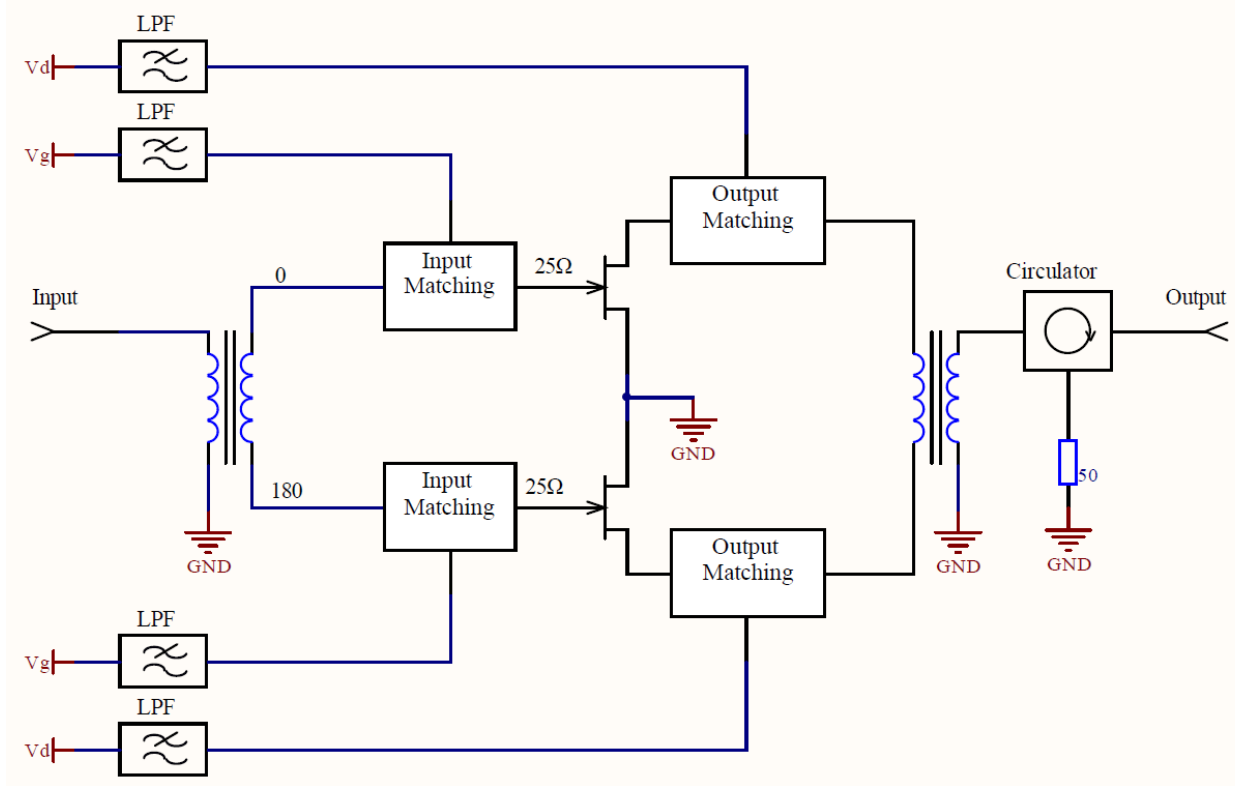

Fig. 1. Block diagram of the push-pull topology of the PA.

phase difference of 180 degrees. A circulator located at the output of the power amplifier is necessary to reduce the influence of the antenna-feeder path on the output matching circuit of the power amplifier.

The next step, using the variable load method "Load Pull" on the basis of a non-linear amplifier model, obtained the input and output impedances of the transistor at an output signal power of $10 \mathrm{~W}(40 \mathrm{dBm})$. Based on the impedances obtained from the simulation results, matching circuits were designed.

Figure 2 shows the basic configuration for load pull modeling: input source tuner, output load tuner, T-shaped couplers for supplying voltage bias to the gate and drain.

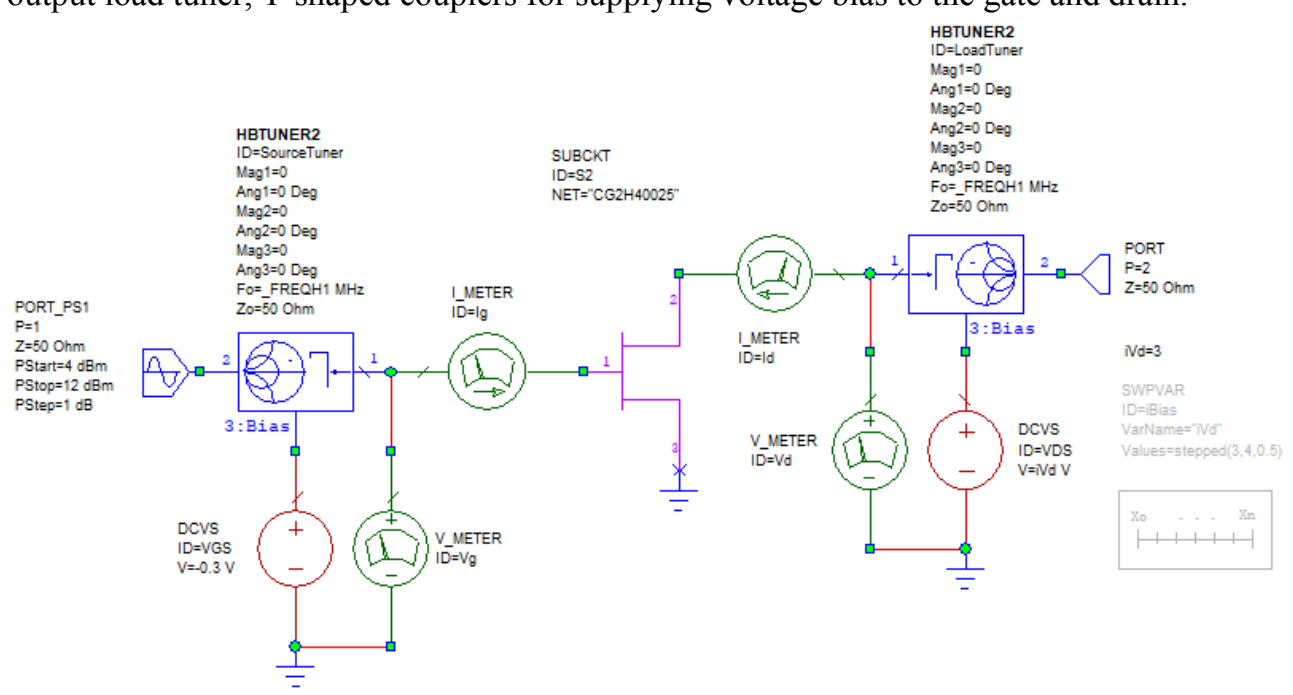

Fig. 2. Load-pull simulation diagram.

Simulation of the source matching scheme was carried out according to the maximum output power, in the case when the output of the device is loaded at $25 \mathrm{Ohms}$. 
The obtained values of the load and source impedance after simulation for the frequency of the main signal $3950 \mathrm{MHz}$ are:

Zsource $=1.2-\mathrm{j} * 8.1$

Zload $=3.8-\mathrm{j} * 0.2$.

At the next stage, the electromagnetic simulation of matching circuit was performed in order to assess the influence of heterogeneity of microstrip transmission lines, according to the results of which the input and output matching circuits were adjusted. Matching circuits were designed based on the conditions for converting the load and source impedance to 25 Ohms and bandwidth requirements. Also, an RC circuit is additionally placed in the input matching circuit to achieve transistor stability when designing matching circuits, based on recommendations made by Cree.

Matching block diagram is shown in Figure 3.

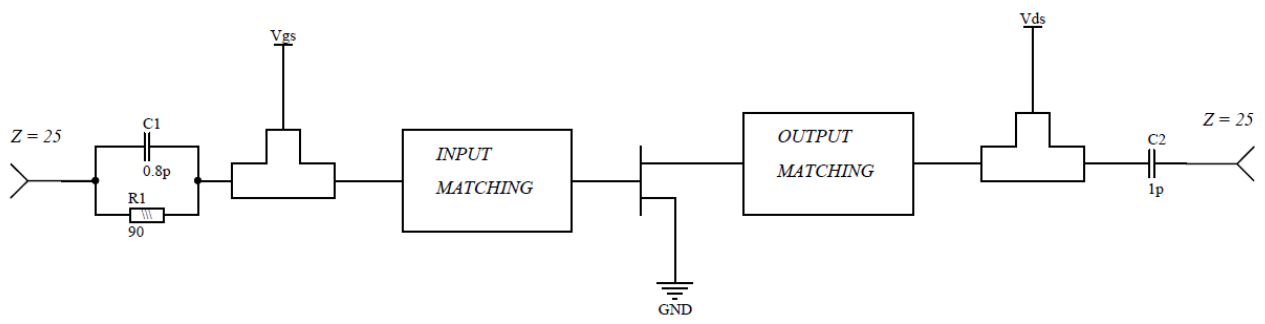

Fig. 3. Block diagram of matching the amplifier stage.

The input matching circuitry contains a serially connected T-shaped power injector made in the form of a $\lambda / 4$ microstrip line and a circuit for converting an impedance of 25 Ohms into a complex conjugate value of the impedance of the $Z_{\text {source }}$.

The output circuit is similar to the input circuit: an impedance conversion circuit is connected in series from the complex conjugate value of the load impedance $Z_{\text {load }}$ and $T$ shaped power injector made in the form of a $\lambda / 4$ microstrip line.

Figure 4 shows the developed input matching circuit diagram.

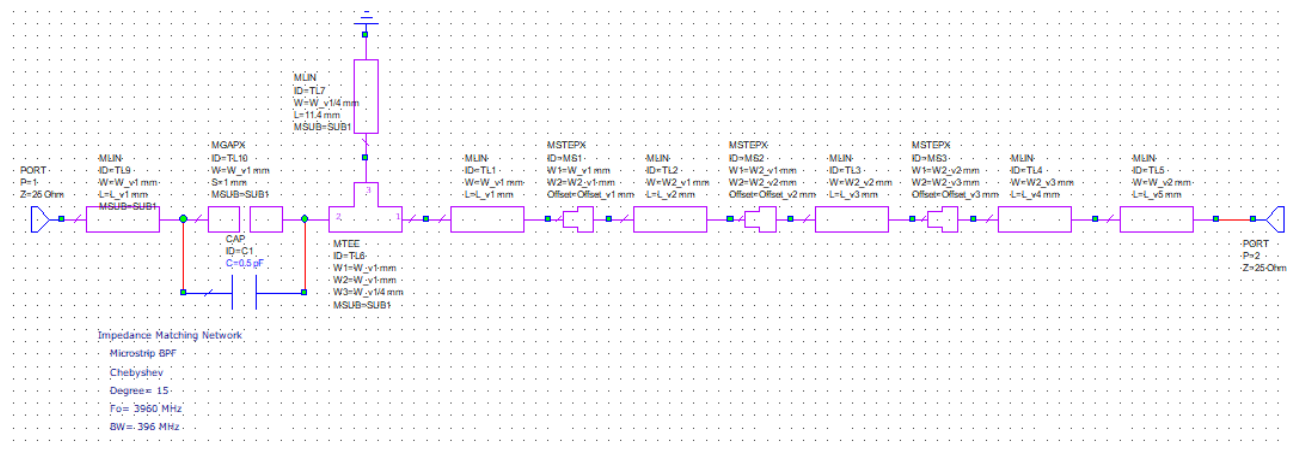

Fig. 4. Electrical circuit of input matching the amplifier stage.

Figure 5 shows the designed electrical circuit of output matching circuit.

After the electromagnetic simulation was made, the matching circuits were adjusted in the way to adjust the input and output impedance values of 25 Ohms. Figure 6 shows the impedance values of the designed input and output matching circuits.

As a signal division / addition scheme, a $\lambda / 4$ coaxial transmission line was used (Figure 7). The use of such line allows to reduce the cost of final product and to simplify the topology of the amplifier. 

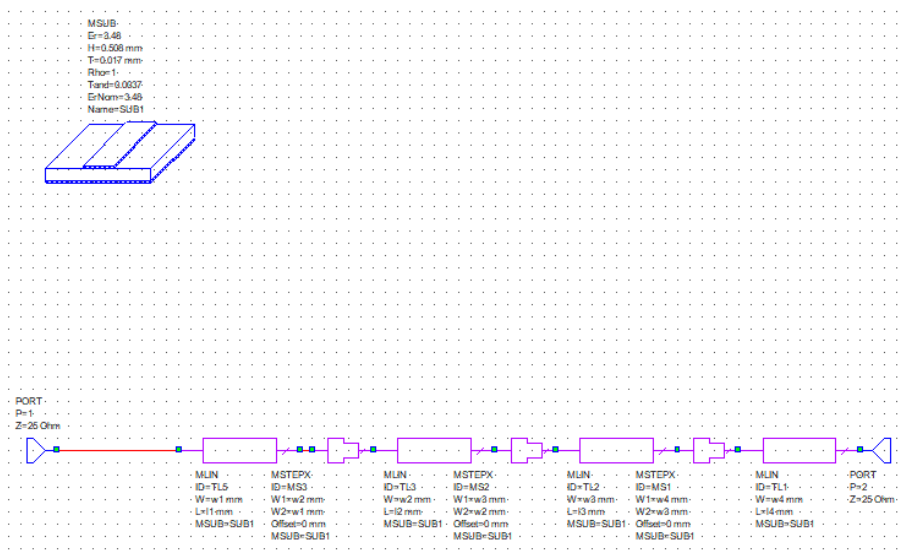

Fig. 5. Electrical circuit of output matching the amplifier stage.

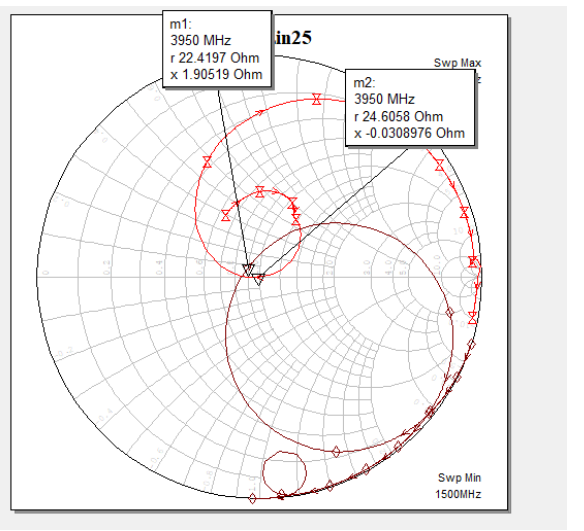

Fig. 6. Impedance of the designed input and output matching circuits after adjustment according to the results of electromagnetic simulation.
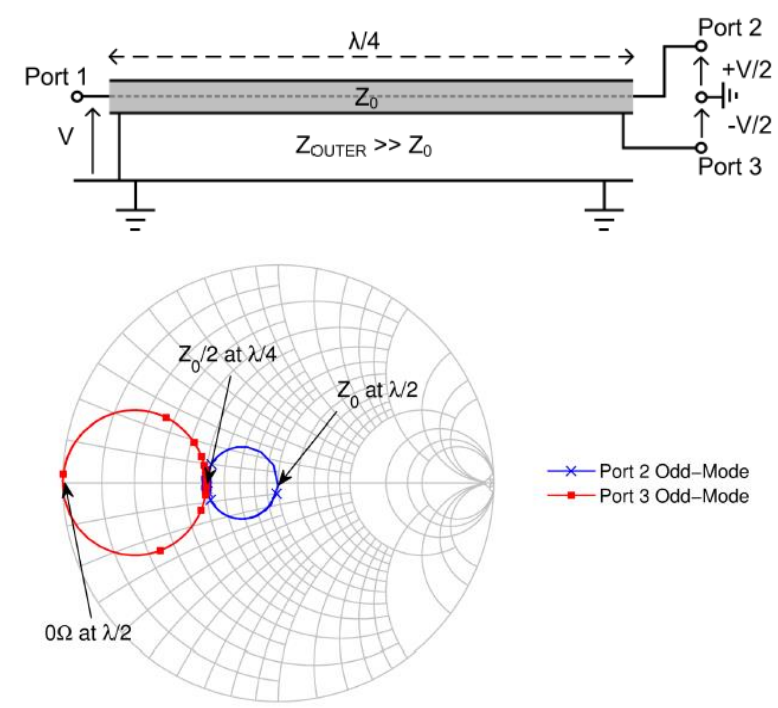

Fig. 7. Signal division / addition scheme based on the $\lambda / 4$ coaxial line and Smith diagram of the simulation results of the circuit [4]. 
Figure 8 shows the resulting final topology of the printed circuit board of the power amplifier unit after adjustments to the input and output matching circuits.

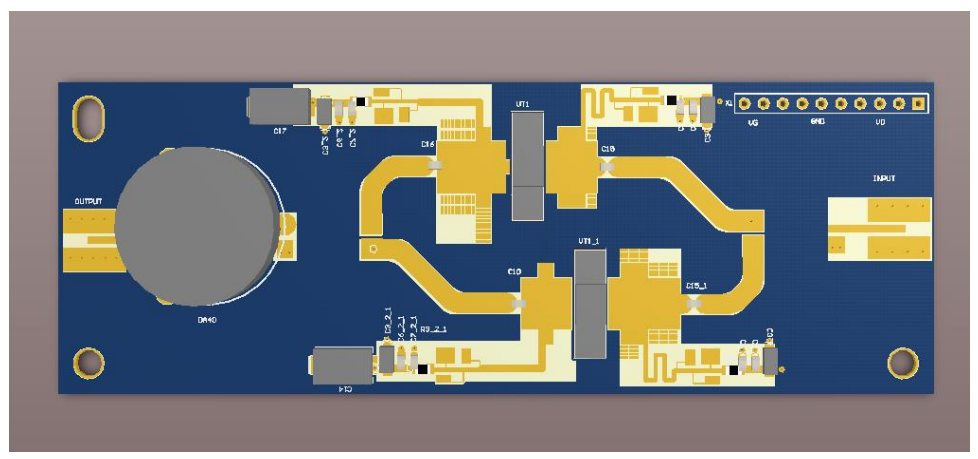

Fig. 8. Topology of the developed push-pull power amplifier on a printed circuit board.

After manufacturing, the characteristics of the push-pull PA were measured.

Table 1 shows the comparative characteristics measured for a push-pull PA and for a Class A PA.

Table 1. Comparative analysis of the manufactured push-pull and class A PA.

\begin{tabular}{|l|c|c|}
\hline Prameter Name & Value for Push Pull PA & Value for Class A PA \\
\hline Frequency range, MHz & $3700-4200$ & $3850-4000$ \\
\hline Gain & 13,5 & 14 \\
\hline $\begin{array}{l}\text { Frequency response ripple in the } \\
\text { operating frequency range, dB }\end{array}$ & 1 & 1 \\
\hline Psat, W & 40 & -20 \\
\hline $\begin{array}{l}\text { Harmonic Level at Psat Power, } \\
\text { dBc }\end{array}$ & -45 & $47 \%$ \\
\hline PAE & $67 \%$ & \\
\hline Dimensions, mm & \multicolumn{2}{|c|}{$115 \times 41 \times 20$} \\
\hline
\end{tabular}

\section{Conclusion}

A methodology is proposed for designing a broadband power amplifier for large signals based on the GaN component base for high-performance nonlinear junction devices, which allows achieving the required characteristics in terms of efficiency, output power, frequency response uniformity, and harmonic signal suppression coefficient (up to minus $45 \mathrm{dBc}$ ) for a wide operating frequency range in small dimensions and at low cost.

The results shown in Table 1 demonstrates that in comparison with Class A amplifiers, the amplifiers made by the Push-Pull topology achieves a wider bandwidth and significantly better harmonic suppression. This improves the performance of nonlinear junction detection devices using a broadband modulated probe signal.

\section{References}

1. O.V. Borisov, A.M. Zubkov, K.A. Ivanov, V.M. Minnebaev, A.V. Red'ka Broadband 70-Watt GaN X-band Power Amplifier. Electronic equipment. Series 2. Semiconductor devices. Issue 2 (233) (2014)

2. Kolomeytsev V. A. Features of power supply of powerful X-band amplifiers in a monolithic design. Solid state electronics. Complex functional blocks of REA. Materials of the IX scientific and technical conference. Zvenigorod, p. 90-92 (2010) 
3. R. M. Smith, J. Lees, P. J. Tasker, J. Benedikt and S. C. Cripps. A 40W Push-Pull Power Amplifier for High Efficiency, Decade Bandwidth Applications at Microwave Frequencies (2012)

4. R. M. Smith, J. Lees, P. J. Tasker, J. Benedikt, and S. C. Cripps. A novel formulation for high efficiency modes in push-pull power amplifiers using transmission line baluns," Microwave and Wireless Components Letters, IEEE, vol. 22, no. 5, pp. 257 259, (May 2012) 